\title{
Edge detection and noise removal by use of a partial differential equation with automatic selection of parameters
}

\author{
CÉLIA A.Z. BARCELOS ${ }^{1 *}$, MAURÍLIO BOAVENTURA ${ }^{2 * *}$ \\ and EVANIVALDO C. SILVA JR. ${ }^{3}$ \\ ${ }^{1}$ FACOM-UFU - Uberlândia, MG and CAC-UFG - Catalão, GO, Brazil \\ 38400-902 Uberlândia, MG, Brazil \\ ${ }^{2}$ DCCE-IBILCE-UNESP, 15054-000 São José do Rio Preto, SP, Brazil \\ ${ }^{3}$ UNIFEV - Votuporanga, SP and FATEC - São José do Rio Preto, SP \\ 15050-500 São José do Rio Preto, SP, Brazil \\ E-mails: celiazb@ufu.br/maurilio@ibilce.unesp.br/evanivaldojr@hotmail.com
}

\begin{abstract}
This work deals with noise removal by the use of an edge preserving method whose parameters are automatically estimated, for any application, by simply providing information about the standard deviation noise level we wish to eliminate. The desired noiseless image $u(x)$, in a Partial Differential Equation based model, can be viewed as the solution of an evolutionary differential equation $u_{t}(x)=F\left(u_{x x}, u_{x}, u, x, t\right)$ which means that the true solution will be reached when $t \rightarrow \infty$. In practical applications we should stop the time " $t$ ", at some moment during this evolutionary process. This work presents a sufficient condition, related to time $t$ and to the standard deviation $\sigma$ of the noise we desire to remove, which gives a constant $T$ such that $u(x, T)$ is a good approximation of $u(x)$. The approach here focused on edge preservation during the noise elimination process as its main characteristic. The balance between edge points and interior points is carried out by a function $g$ which depends on the initial noisy image $u\left(x, t_{0}\right)$, the standard deviation of the noise we want to eliminate and a constant $k$. The $k$ parameter estimation is also presented in this work therefore making, the proposed model automatic. The model's feasibility and the choice of the optimal time scale is evident through out the various experimental results.
\end{abstract}

\#606/04. Received: 26/V/04. Accepted: 09/XI/04.

*Supported by CNPq - Project 302549

**Partially supported by CAPES 


\section{Mathematical subject classification: $94 \mathrm{~A} 08,68 \mathrm{U} 10,68 \mathrm{U} 20$.}

Key words: image processing, noise removal, edge detection, diffusion equation.

\section{Introduction}

Over the past few years the use of partial differential equations (PDE) in image modelling denoising and edge-detection has grown significantly. The PDE based models modify an image, a curve or a surface with a PDE by looking for its solution. This theory had it's beginning with the formulation proposal of Marr and Hildreth [8] with the definition of optimal filter smoothing using the Gaussian nucleus, and consequently the use of the heat equation, for the smoothing of the images which brings about the noise elimination. Starting from the mentioned formulation, other models were presented in associated literature and through increments or alterations to the original structure of the heat equation where produced visibly superior results ([1], [7], [11], etc.). A fact common to all these models, be they isotropic or anisotropic, is the generation of data structures that allow us to consider the evolution concept as a time parameter denominated as space-scale. These structures, which are called Scale Space, are of dynamic character because they establish the evolution of PDEs on the " $t$ " - scale, representing the image $u(x, t)$ at multiple scales $t$. The contribution presented in [6] and [15] introduced the image representation obtained by Gaussian filtering in space scale context. The Gaussian space scale and the space scale given by the non linear partial differential equations for the noise elimination and segmentation process are presented in this paper.

In general there are parameters in the continuous formulation of these models. To solve these models numerically, it has become necessary to attribute real values to these parameters, and frequently, they are taken with values that produce the best results from the visual point of view ([4], [11] and [12]). It is not an easy task to solve, as the parameters depend on, for example, the initial image to be processed and the amount of noise that we desire to remove. The desired noiseless image $u(x, t)$, in an PDE based model, can be viewed as the solution of an evolutionary differential equation which means that the true solution will be reached when $t \rightarrow \infty$. In practical applications we should stop 
the time " $t$ "' at some moment during this evolutionary process. This work deals with the relationship between the optimal time of suavization, which gives an estimate for the stop time of the evolutionary process, and the level of noise elimination desired. The viability of finding an ideal stop evolutionary time for the differential equation avoids insufficient or surplus computation time. The insufficient computation time will not give better results and the evolution of the differential equations beyond the necessary smoothing time causes an unnecessarily high computational cost. The stop time concept gives an estimative for the time which the scale evolves in order to guarantee efficiency and computational gain in the denoising process of an image. Motivated by experimental results and based on analysis of the diffusion term of a non linear partial differential equation model we introduced a sufficient condition, related to time $t$ and with the standard deviation of the noise $\sigma$, which gives a constant $T$ such that $u(x, T)$ is a good approximation of the true solution $u(x, t)$. We also present the parameter estimation for the selected PDE model therefore making, the proposed model automatic for any application by simply providing information about the standard deviation noise level we wish to eliminate. The model's feasibility and the choice of the optimal time scale is evident through the various experimental results.

The paper is organized as follows. In Section 2, three PDE based models, involving parameters in their formulation are presented for reconstruction of image $u$. Section 3 presents the Gaussian space-scale concepts. In Section 4 a non linear space-scale is presented. The optimal time concept, which permits us to estimate the stop time parameter, is introduced in section 5. In Section 6 the approximation for parameter $k$ is presented, and the experimental results are presented in Section 7. Finally the paper is concluded in Section 8.

\section{Image denoising}

In this paper, we consider an image to be a real value function on a spatial domain $\Omega, u: \Omega \subset \mathbb{R}^{2} \rightarrow \mathbb{R}$. Let $I$ be the intensity of an image obtained from a noiseless image by adding Gaussian noise with zero mean, defined on a rectangle $\Omega \subset \mathbb{R}^{2}$ called image support. We assume here that the degradation model is of the form: $I=u+\eta$, where $\eta$ represents the noise in the image. 
Several techniques have been proposed for the reconstruction of $u$ from $I$. One of them is the Total Variation method proposed by Rudin, Osher and Fatemi [9] which consists of minimizing the following functional:

$$
E_{T V}(u)=\int_{\Omega}\left(|\nabla u|+\frac{\beta}{2}|u-I|^{2}\right) d x,
$$

where $\beta$ is a positive parameter. The solution was obtained by finding a steady state solution of a time dependent partial differential equation, which is the evolution of the Euler-Lagrange equation for $E(u)$. This means that they solved

$$
\begin{gathered}
u_{t}=\operatorname{div}(\nabla u /|\nabla u|)-\beta(u-I), \\
u(x, 0)=I(x), \\
\left.\frac{\partial u}{\partial n}\right|_{\partial \Omega \times R_{+}}=0 .
\end{gathered}
$$

The constant $\beta$ is given by:

$$
\beta=-\frac{1}{2 \sigma^{2}} \int_{\Omega}\left[|\nabla u|-\frac{\nabla I . \nabla u}{|\nabla u|}\right] d x
$$

In [13] a closely related problem is proposed. The denoising problem is solved by the minimization of

$$
f(u)=\frac{1}{2}|u-I|^{2}+\alpha J_{\beta}(u),
$$

where

$$
J_{\beta}(u)=\int_{\Omega}\left(\left|\nabla u^{2}\right|+\beta^{2}\right) d x,
$$

$\alpha$ and $\beta$ being positive parameters. The parameter $\alpha$ in (2.4) is inversely proportional to the Lagrange multiplier $\beta$ given in (2.1). This approach is referred to as Tikhonov reguralization. The authors present a numerical study of the $\alpha$ and $\beta$ parameters effects.

These models were obtained from variational problems, therefore the evolutionary equations do not necessarily need to be obtained from the energy functional, as with the equation:

$$
u_{t}=|\nabla u| \operatorname{div}\left(\frac{\nabla u}{|\nabla u|}\right)
$$


known as the mean curvature flow equation, which has inspired many other models because of its geometric interpretation. The existence, uniqueness and stability is due to the work of L.C. Evans [5]. As this equation is not able to preserve the edges localization, Alvares, Lions and Morel proposed in [1] the following non linear parabolic equation for noise removal

$$
u_{t}=g\left(\left|\nabla G_{\sigma} * u\right|\right)|\nabla u| \operatorname{div}\left(\frac{\nabla u}{|\nabla u|}\right),
$$

where $u(x, 0)=I(x)$ represents the initial noisy image, $g\left(\left|\nabla G_{\sigma} * u\right|\right)$, is a smooth non-increasing function with $g(0)=1, g(s) \geq 0$, and $g(s) \rightarrow 0$ as $s \rightarrow \infty$. The usual choice for $g$ takes the form $g(s)=\frac{1}{\left(1+k s^{2}\right)}$ with $k$ as a parameter. In the formula of most of the models based on PDE there exist parameters of which in some cases are determined by specific procedures and in others, for example [3], the parameters are taken from that which produces the best results.

These noise elimination processes reach their highest performance according to the $t$ evolution on the scale. Considering all $t \in(0, T]$ we have a sequence of noisy approximations of the original image which have a non-increasing level of noise, generating in this way a temporal space scale [14] and [15].

\section{The Gaussian space scale}

The PDE models in image processing act in a way as to construct a sequence of images $u\left(x, t_{n}\right)$ at time $t_{n}$ starting with the initial image $u(x, 0)$ and where each one of these images represents an approach for the solution of the equation. Consequently, when we refer to "time" $t$, we are refereing to a certain stage of the reconstruction process of an image. The noise elimination process is based on a filtering procedure. The function commonly used for this purpose is the Gaussian function of variance $\sigma^{2}$ which is given by

$$
G_{t}(x)=\frac{1}{\sqrt{4 \pi t}} e^{-x^{2} \frac{1}{4 t}}, \quad \text { where } t=\frac{\sigma^{2}}{2}
$$

and the variance $\sigma$ is the size of the filter. The Gaussian $n$-dimensional signal space scale is defined as the composition of this signal with all possible variance Gaussian functions, i.e., making $t \rightarrow \infty$. 
As the Gaussian function is the solution of the heat equation, the Gaussian space scale can be formally defined as:

Definition 3.1. Given an image $I(x): \mathbb{R}^{2} \rightarrow \mathbb{R}$, the Gaussian space scale for $u(x)$ is defined as a function $u: \mathbb{R}^{2} \times \mathbb{R}_{+} \rightarrow \mathbb{R}$ (denoted by $u(x, t)$, where $\left.t \in \mathbb{R}_{+}\right)$which is the solution of the heat equation:

$$
\begin{gathered}
\frac{\partial}{\partial t} u(x, t)=\Delta u(x, t), \\
u(x, 0)=I(x) .
\end{gathered}
$$

The processing of an image by the heat equation will flatten everything in the image, losing all important structures of the image (Fig. 3.1.). That effect is due to the fact that the Gaussian filter has assumed the coefficient of conductance as constant [6] and [7].
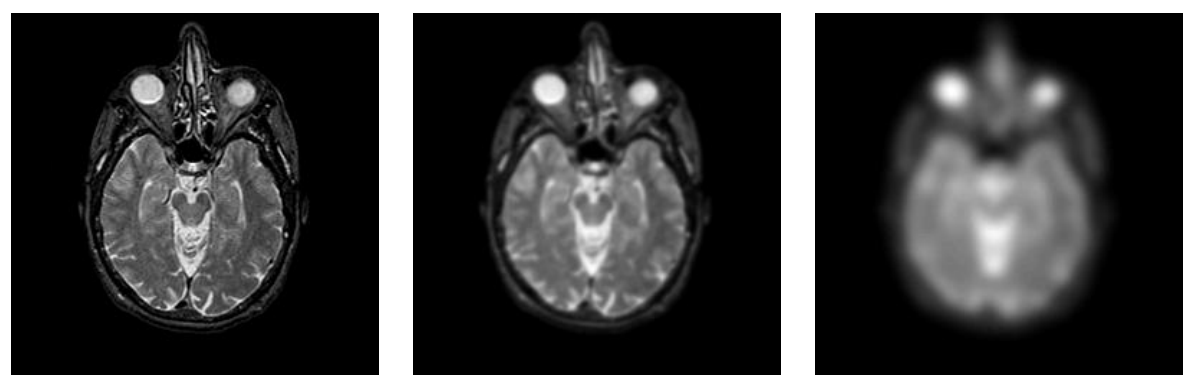

Figure 3.1 - Brain image and two suavization versions obtained by Gaussian filters with different sizes.

The solution of (3.1)-(3.2) is given by the convolution of the image $I(x)$ with the Gaussian function $G_{\sigma}$, i.e., $u(x, t)=G_{t}(x) * I(x)$. As a consequence the Gaussian scale space obtains the properties: linearity, invariance by translations and causality which means that the signal $u(x, 0)$ is simplified when the scale grows $(t \rightarrow \infty)$. We can observe that the space scale increases by one in the dimension of the data structure. 

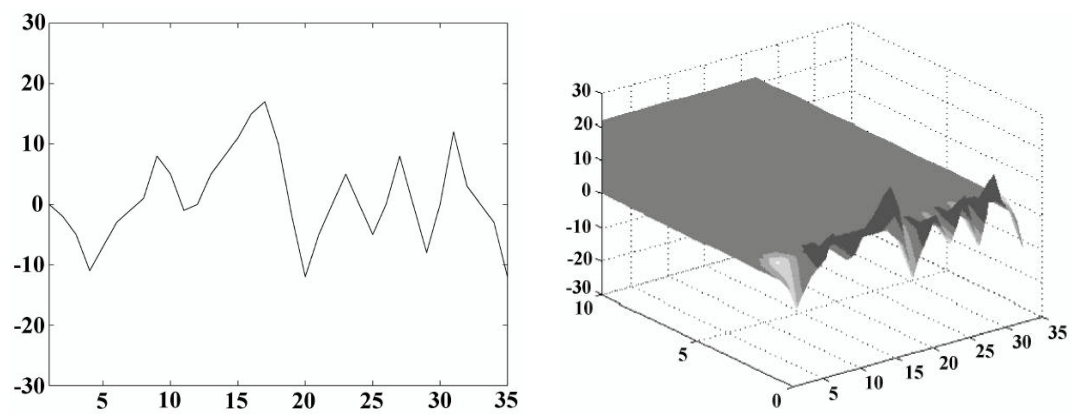

Figure 3.2 - Unidimensional signal and its space scale.
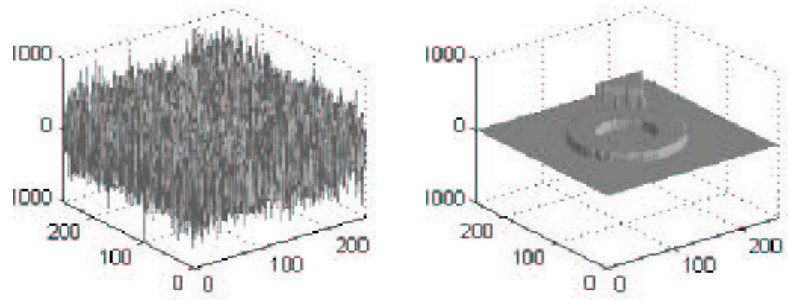

Figure 3.3(a) - Noisy and noiseless bidimensional image signal.
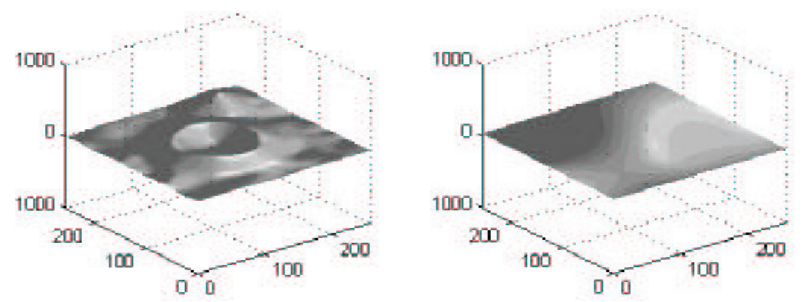

Figure 3.3(b) - Bi-dimensional signal of the Fig. 3.3(a) noisy image processed by the heat equation (displayed at time $t=40$ and $t=966$ ).

\section{Non linear space scale}

The first PDE used for noise removal was the heat equation which when applied to a noisy image $I(x)$ flattens out everything causing the loss of all information. In order to eliminate these inconveniences in relation to the structure loss provoked by the evolution of the heat equation there exist several works which modify the PDE's used. The principal modification is based on the addition of new terms in the equation, or in the modification of those which already exist. As a 
consequence we have the loss of linearity of the non linear scale spaces generated by the PDE model as in the equations (2.1-2.3), (2.6) and (3.1). With the objective of eliminating unnecessary parameters, where in many cases they are co-related we propose in this paper an equation to eliminate noise and detect borders in an image where only two inter related parameters exist, and which depend on the image. The model studied here has as its main characteristic the elimination of the parameters used in our previous work([2]), we were inspired by the works of Rudin, Osher and Fatemi([9]) e Alvares, Lions and Morel ([1]). The enhancement of the initial image $I$ is performed by the following differential equation:

$$
u_{t}=g\left(\left|\nabla G_{\sigma} * u\right|\right)|\nabla u| \operatorname{div}\left(\frac{\nabla u}{|\nabla u|}\right)-(1-g)(u-I),
$$

where $u(x, 0)=I(x)$ represents the initial noisy image, $g\left(\left|\nabla G_{\sigma} * u\right|\right)$, as in [1], is a smooth non-increasing function with $g(0)=1, g(s) \geq 0, g(s) \rightarrow 0$ as $s \rightarrow \infty$. The usual choice for $g$ takes the form $g(s)=\frac{1}{\left(1+k s^{2}\right)}$.

This model allows one to perform selective smoothing in accordance to the size of the image gradient at point $x$. The constant $\sigma$, to be used in $G_{\sigma}$ calculation, is taken as the standard noise deviation of the initial image $I$ and $G_{\sigma}$ is a Gaussian function.

The approach given by equation (4.1) presents as its main characteristic edge preservation during the noise elimination process and can be viewed as the balance between "suavization" and "stay close to I". This balance is carried out by the function $g$, which is taken as

$$
g=g\left(\left|G_{\sigma_{I}} * \nabla u\right|\right)=\frac{1}{1+k\left|G_{\sigma_{I}} * \nabla u\right|^{2}},
$$

$k$ is a $\sigma_{I}$ dependent constant, where $\sigma_{I}$ is the standard deviation of the initial noisy image $I$. The main advantage of this balance is edge preservation. It is easy to notice, by the space scale generated by this model, that the edges are really very well preserved (see figures 7.1, 7.2, 7.3, 7.4, 7.5 and 7.6).

There are only two parameters, in the numerical solution of the model given by the equation (4.1), to be determined: a constant $k$ in (4.2) and the stop for the evolutionary time $t$ known here as the optimal suavization stop time. 

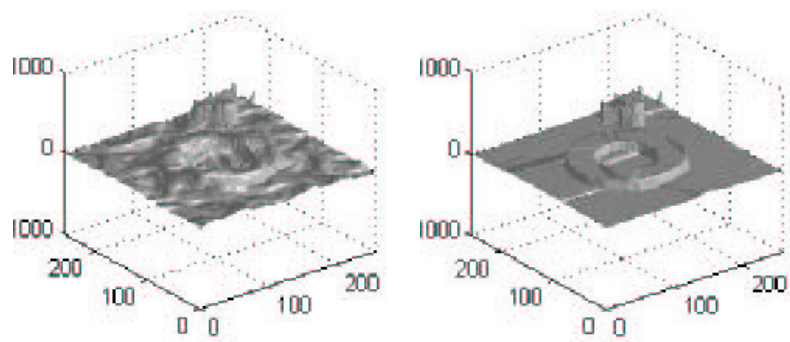

Figure 4.1 - Bi-dimensional signal of the noisy image Fig. 3.3(a) obtained by equation (4.1) at time $t=40$ and $t=966$.
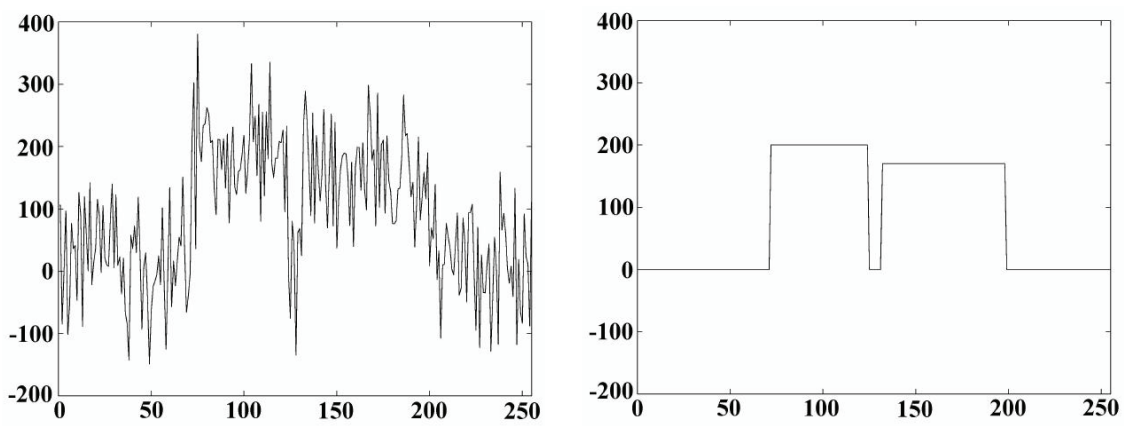

Figure 4.2 - Unidimensional Gaussian noisy $(\mathrm{SNR}=0 \mathrm{db})$ and noiseless signal.
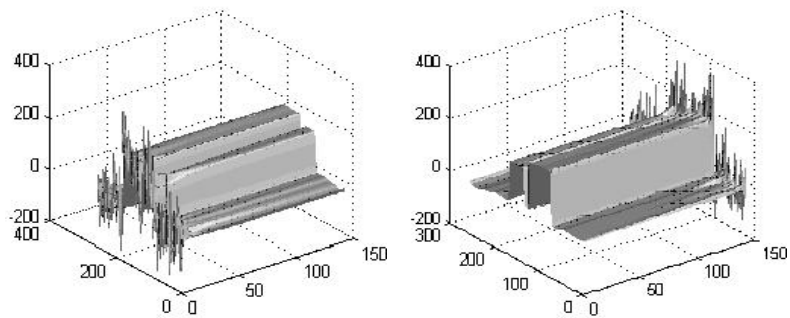

Figure 4.3 - Front and back view of the space scale generated by the equation (4.1) from the noisy image given in Fig. 4.2.

\section{The optimal suavization stop time}

The desired noiseless image $u(x)$ will be the solution of the equation (4.1). That means: we will have the true solution when $t \rightarrow \infty$. We will call the optimal suavization time a constant $T$, such that $u(x, T)$ is a good approximation of $u(x, t \rightarrow \infty)$. 
Here is presented a definition for the optimal suavization "time" as the time $T>0$ sufficient to reduce the noise of an image $u\left(x, t_{0}\right)$ to acceptable levels.

Definition 5.1. Given a small constant $\varepsilon>0$ and an image $u\left(x, t_{0}\right)$, the time $T(\varepsilon)>0$ is the said optimal suavization time when $\left|u(x, T)-u\left(x, t_{s}\right)\right| \leq \varepsilon$, for all $t_{s}>T$.

Although the introduction of the forcing term $(u-I)$ eliminates the real necessity for the definition of the stop time in the evolutionary process given by the PDE (2.1), in practical application, we must stop the evolutionary process at a certain moment in the scale where an arbitrary choice of the constant $T$ would not be satisfactory.

If we interrupt the process before the necessary time $T$, we will have a non satisfactory smoothed image. On the other hand, if we apply the model beyond the time $T$ it means an undesirable additional computational cost.

If $\varepsilon$ is small enough, the optimal suavization time is the time $T$ which produces a satisfactory level of suavization in an image $u\left(x, t_{0}\right)$, it means that from the perceptive point of view all the images $u\left(x, t^{\prime}\right)$ obtained at scales $t^{\prime}$ greater than $T$ have not been substantially changed.

Taking into account that the diffusion in the model (4.1) is performed by the term $|\nabla u| \operatorname{div}\left(\frac{\nabla u}{|\nabla u|}\right)$, (which is presented in the mean curvature flow equation) and for the elimination, in an interval of time $[0, T]$, the noise presented in a given initial image with standard deviation $\sigma_{I}$ in $t=0$, we should suppose that this interval of time $[0, T]$ will be sufficient for the model based on the curvature equation to make a circle of ratio $R(0)$ disappear.

As the circle's boundary moves at velocity equal to the curvature, which in turn is the inverse of the ratio $R(t)$, we have the following equation:

$$
\frac{d R(t)}{d t}=-\frac{1}{R(t)}
$$

With the goal of preserving important details we will distinguish the image processing time with different standard deviations. Images with lower standard deviation should be processed slower than those which present greater standard deviation. To reach our objective we will use the standard deviation of the initial image as a determining factor to control the contraction speed of the radius $R(t)$. 
Motivated by these factor, and which have been proven experimentally, and, also considering definition 2, we propose, in the next theorem, an estimate for the ideal $T$ time to stop the evolutionary process given by model (4.1).

Theorem 5.1. The time $T$ sufficient for the model (4.1) to give a satisfactory level of suavization when applied in a given initial noisy image $I(x)=u\left(x, t_{0}=\right.$ $0)$, is given by

$$
T=\frac{\sigma^{2}}{a} .
$$

where $\sigma$ is the standard deviation of the noise in $I(x), \sigma_{I}$ is the standard deviation of the noisy image $I(x)$ and $a=2 \sigma_{I}$ is the constant present in the Gaussian nucleus

$$
G_{t}(x)=\frac{1}{a \pi t} e^{-|x| / a t}, x \in \mathbb{R}^{n} .
$$

Proof. Let $\sigma(t)$ be the function that gives, for each $t$, the value of the standard deviation of the noise present in $u(x, t)$, in this way we have $\sigma(0)=\sigma_{I}$. Considering $\sigma(0)$ as the initial ratio of a circle, we have that $\sigma(t)$ is related to motion by mean curvature flow equation in the same way as $R(t)$. It means that $\sigma(t)$ goes to zero when $R(t)$ goes to zero. As the speed of a circle's boundary contraction can be modified by a factor, the equation (5.1) can be reformulate as

$$
\frac{d \sigma(t)}{d t}=-\sigma_{I} \frac{1}{\sigma(t)}
$$

where $\sigma_{I}$ determines the contraction velocity of $\sigma(t)$. Solving this equation, we have

$$
\sigma_{I} T=\frac{1}{2}\left(-\sigma^{2}(T)+\sigma^{2}(0)\right)=\frac{\sigma^{2}(0)}{2} .
$$

Then, if

$$
T=\frac{\sigma^{2}(0)}{2 \sigma_{I}}=\frac{\sigma^{2}}{2 \sigma_{I}}
$$

the standard deviation $\sigma(t)$ will be zero as a consequence of the $T$ construction. 
This theorem says from the theoretical point of view, that the optimal time $T$ is the time in which the desired amount of noise elimination is reached, this means the $u(x, T)$ is a denoised image approximation of the $u(x, 0)$, excepted on the edge points where the smoothing by the diffusion term was not performed.

As the proposed model is building with respect to preserving edges, then the obtained image reaches its stationary state point, preserving the edges initial characteristics. For each $t_{s}>T$, the image $u\left(x, t_{s}\right)$ is close to $u(x, T)$, this means $\left|u(x, T)-u\left(x, t_{s}\right)\right|<\varepsilon$ for small $\varepsilon$.

Corollary 5.1. Let $t_{r}, t_{s}>T$. Then $\left|u\left(x, t_{r}\right)-u\left(x, t_{s}\right)\right|<\varepsilon$, where $u\left(x, t_{r}\right)$ and $u\left(x, t_{s}\right)$ are two approximations of the image $u(x)$ obtained by the model (4.1) in different scales, $t_{r}$ and $t_{s}$, with the same initial condition $u(x, 0)$.

Proof. Let $t_{r}$ and $t_{s}$ constants greater than $T$, and $u\left(x, t_{r}\right)$ and $u\left(x, t_{s}\right)$ the images obtained at the scale $t_{r}$ and $t_{s}$, respectively. According to Theorem 5.1, there exists a constant $\varepsilon_{1}$, such that:

$$
|u(x, T)-u(x)|<\varepsilon_{1} .
$$

Considering $\left|u(x, T)-u\left(x, t_{i}\right)\right|$ for any $t_{i}>T$, we have

$$
\begin{aligned}
\left|u(x, T)-u\left(x, t_{i}\right)\right| & \leq|u(x, T)-u(x)|+\left|u(x)-u\left(x, t_{i}\right)\right| \\
& \leq 2|u(x, T)-u(x)|<2 \varepsilon_{1}=\varepsilon_{i}^{\prime},
\end{aligned}
$$

choosing $\varepsilon^{\prime}=\max \left\{\varepsilon_{i}^{\prime}\right\}, \quad i=r, s$, follows that,

$$
\left|u(x, T)-u\left(x, t_{i}\right)\right|<\varepsilon^{\prime}, \quad i=r, s .
$$

In this way

$$
\begin{aligned}
\left|u\left(x, t_{r}\right)-u\left(x, t_{s}\right)\right| & =\left|u\left(x, t_{r}\right)-u\left(x, t_{s}\right)+u(x, T)-u(x, T)\right| \\
& \leq\left|u(x, T)-u\left(x, t_{r}\right)\right|+\left|u(x, T)-u\left(x, t_{s}\right)\right|<2 \varepsilon^{\prime} \\
& =\varepsilon .
\end{aligned}
$$




\section{The $k$ parameter}

At this moment, there is only one unknown parameter in the model (4.1) presented in the function $g$ defined by the equation (4.2). This parameter, $k$, is frequently taken as an arbitrary parameter chosen by the user and is intrinsically related to the amount of detail we want to preserve. As a consequence this constant works as the parameter for edge selection. For a fixed image, as the $k$ increases, spurious edges will be identified which means that for small values only well defined edges will be selected, in other words those edges with a strong intensity level contrast.

The success of the model depends on the right determination of the edges which depends on the adequate choice for the constant $k$, which can cause damage if the user is inexperienced.

In an attempt to avoid an inadequate choice for this constant, based on the experimental data, and in the verification of the fact that the constant $k$ should vary with the amount of the noise presented in an image, we present a model to calculate the constant $k$.

The analysis of the dispersion diagram of $k$ versus $\sigma_{I}$, values carried out in diverse experiments (where $k$ was obtained experimentally as that which supplies the best result from a visual point of view), we find that the curve which adapts best to the data is the exponential type, which is $k\left(\sigma_{I}\right)$ of the type $a e^{b s}$. It was therefore checked through the many tests carried out that the behavior of the curve which represents these points dispersal (Fig. 6.1(a)), presents a gap within the point's neighborhood which represents the standard deviation of the initial image at approximately 200. In this way we propose a domain division of the selected data, selecting representative tests from the image, each of different nature and complexities, with Gaussian noise of different levels, obtaining an approximation function given by:

$$
k\left(\sigma_{I}\right)= \begin{cases}1277.175 e^{0.040104385 \sigma_{I}}, & \text { if } \quad 0 \leq \sigma_{I} \leq 201 \\ 2429901.476 e^{0.004721247 \sigma_{I},} & \text { if } \quad 201<\sigma_{I} \leq 350\end{cases}
$$

using the least square method.

The quality of the approximation can be validated by the plots Fig. 6.1(a-b). 


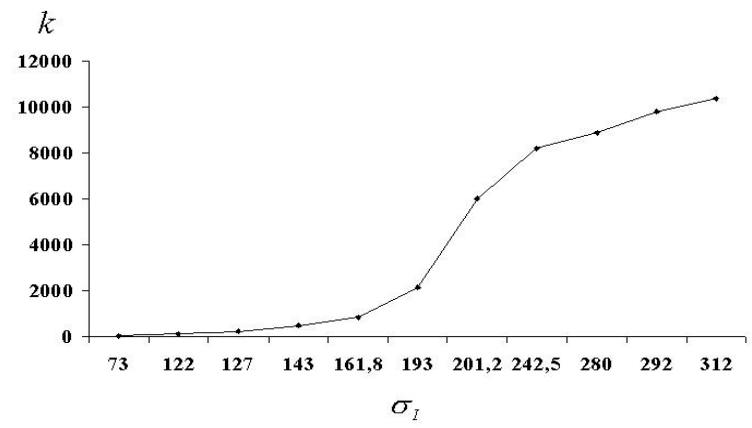

Figure 6.1(a) - Graph showing the parameters $k$ related to $\sigma_{I}$ tabled values $\left(\sigma_{I}\right.$ $\in[0,350])$.
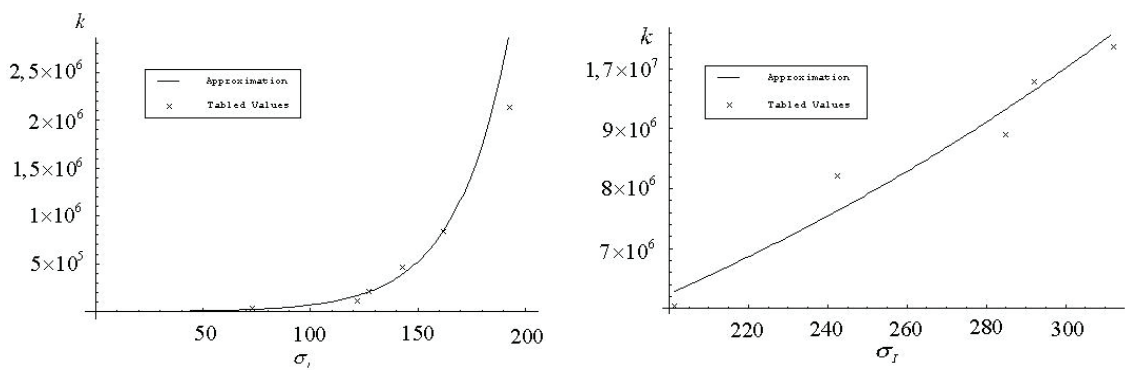

Figure 6.1(b) and (c) $-k\left(\sigma_{I}\right)$ graphs $\left(\sigma_{I} \in[0,201]\right.$ and $\sigma_{I} \in[201,350]$, respectively).

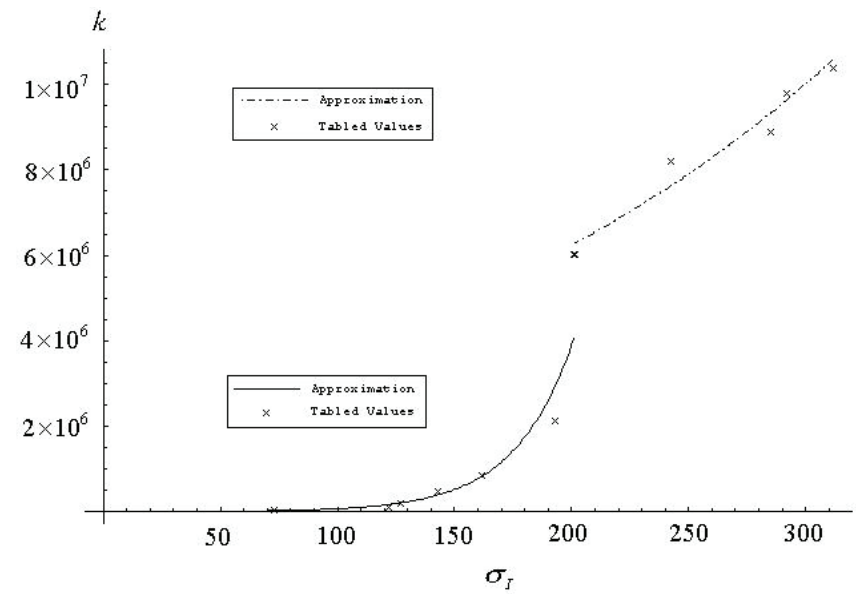

Figure 6.1(d) - The $k\left(\sigma_{I}\right)$ function graph $\left(\sigma_{I} \in[0,350]\right)$. 
The ends of the interval $[0,350]$ are related to $15 \mathrm{db}$ and $-15 \mathrm{db}$ noise levels, respectively. This interval gives the range of the adequate noise presented in an image to be processed using (6.1) to determine the constant $k$. The results are very good in spite of the range of the noise interval being very large when compared with the number of points used to obtain the function $k\left(\sigma_{I}\right)$. Various tests were carried out on images with different levels of Gaussian noise and different levels of complexity, which showed the effectiveness of the equation (6.1).

\section{Experimental results}

In this section, we present in figures 6-8 the results obtained by applying the model in (4.1) with the selected parameter $k$ using the equation (6.1) and stopping the process at the ideal stop time $T$.

Our test images are represented by $256 \times 256$ matrices of intensity values. We let $u_{i, j}$ denote the value of the intensity of the image $u$ at the pixel $(x=i \Delta t$, $y=j \Delta t)$. We denote $u\left(i, j, t_{n}\right)$ by $u_{i, j}^{n}$.

The time derivative $u_{t}$ at $\left(i, j, t_{n}\right)$ is approximated by the forward difference $\frac{u_{i, j}^{n+1}-u_{i, j}^{n}}{\Delta t}$. The diffusion term

$$
|\nabla u|\left(\operatorname{div}\left(\frac{\nabla u}{|\nabla u|}\right)\right)=\frac{u_{x}^{2} u_{y y}-2 u_{x} u_{y} u_{x y}+u_{y}^{2} u_{x x}}{u_{x}^{2}+u_{y}^{2}}
$$

in (4.1) being approximated using central differences.

Figure 7.1 shows the performance of the model (4.1) in a wall picture with Gaussian noise of different tree levels: 12,6 and $0 \mathrm{db}$ with standard deviation as 28.8, 57.5 and 115.2, respectively. We can easily see in figure 7.1(b) and 7.1(c) the denoised image obtained at time $T$ and in figure 7.1(d) we can see that the noise present in the $130^{\text {th }}$ row was eliminated. Figure 7.2 and figure 7.3 show the results obtained in Lenna and in the textured image, respectively. Figure 7.4 presents a noisy syntectic image with $\sigma_{I}=139.1$ and $\mathrm{SNR}=-3.5 \mathrm{db}$. Figure 7.5 also represents a synthetic image with a very high level of noise ( $\mathrm{SNR}=-12 \mathrm{db})$, with a standard deviation $\sigma_{I}=250$. In our last example we present an image with $7 \mathrm{db}$ Gaussian noise and standard deviation $\sigma_{I}=201$. 

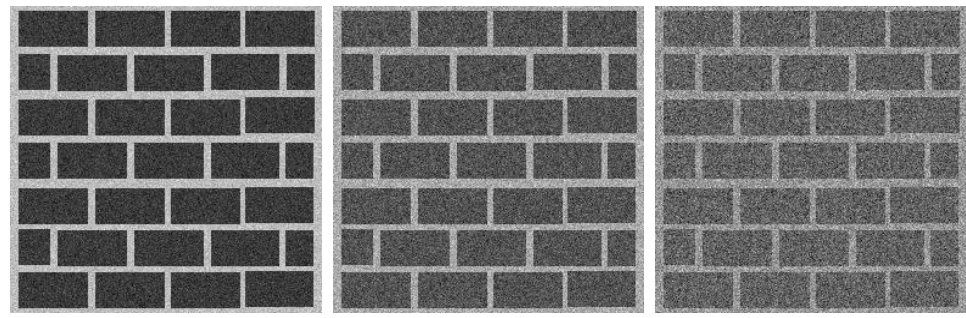

Figure 7.1(a) - Wall noisy image with SNR 12, 6 and 0db, respectively.
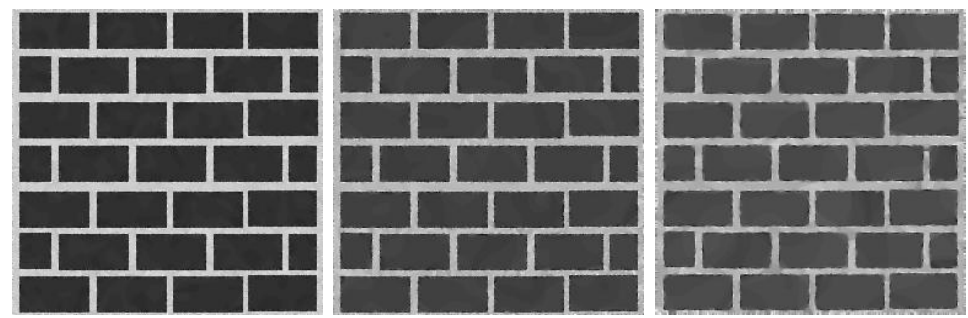

Figure 7.1(b) - Plots of the reconstructed images (Fig. 7.1(a)) at time $\mathrm{t}=T$.
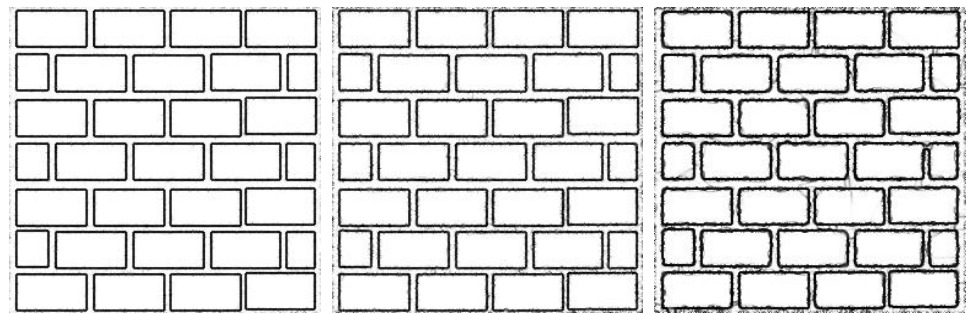

Figure 7.1(c) - Segmentation.
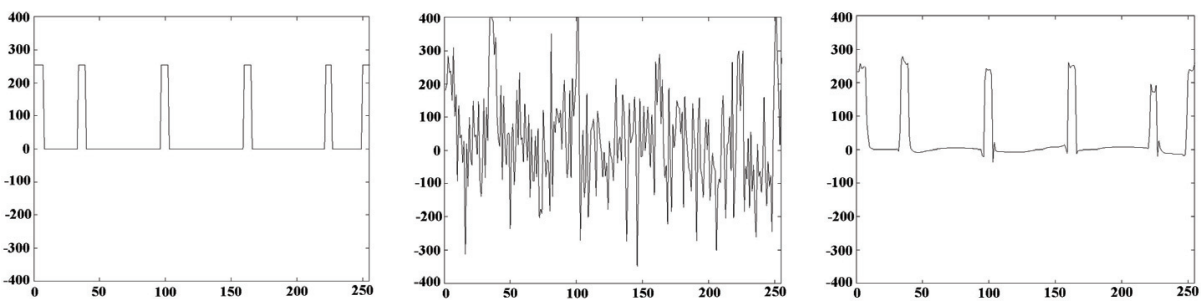

Figure $7.1(\mathrm{~d})$ - Plots of $130^{\text {th }}$ lines of the true, noisy $(\mathrm{SNR}=0 \mathrm{db})$ and reconstructed image, respectively. 

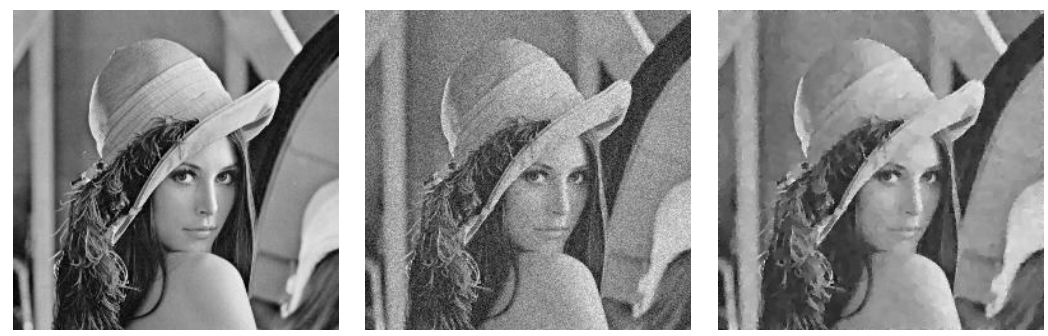

Figure 7.2 - Lenna, noisy SNR=9db and reconstructed image, respectively.
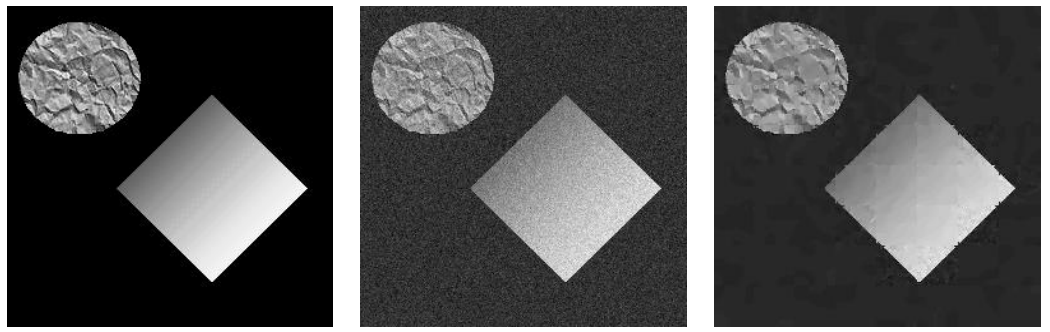

Figure 7.3 - Synthetic textured image, noisy image $(\mathrm{SNR}=12 \mathrm{db})$, and reconstructed image, respectively.
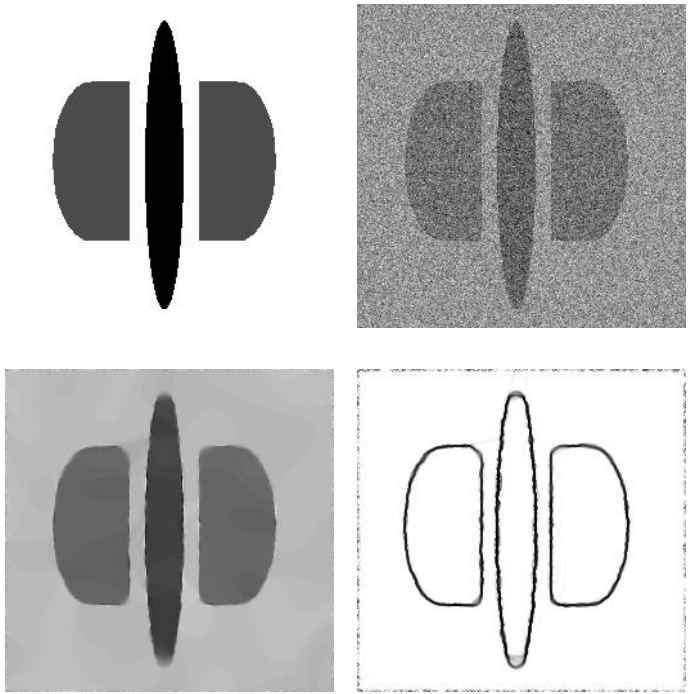

Figure 7.4 - Noiseless, noisy $(\mathrm{SNR}=-3.5 \mathrm{db})$, reconstructed and segmented image (left to right, top to bottom). 

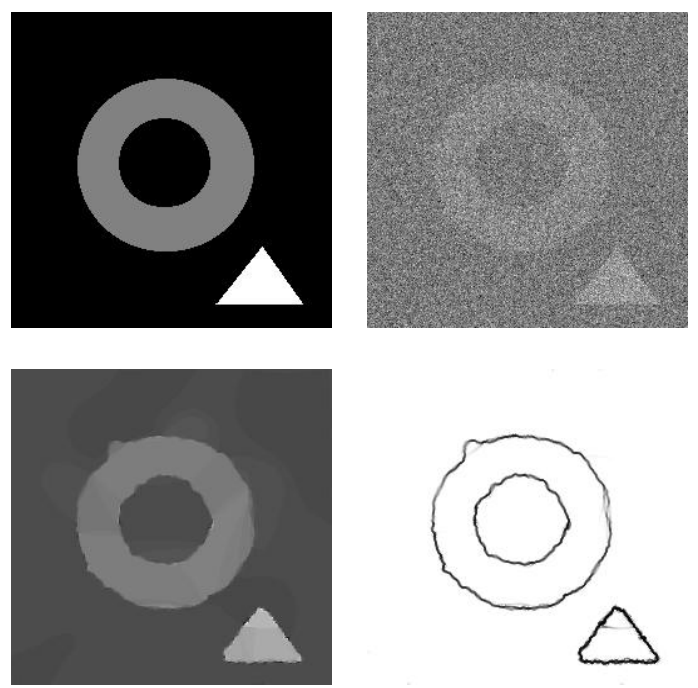

Figure 7.5 - Original, noisy $(\mathrm{SNR}=-12 \mathrm{db})$, reconstructed and segmented image (left to right, top to bottom).
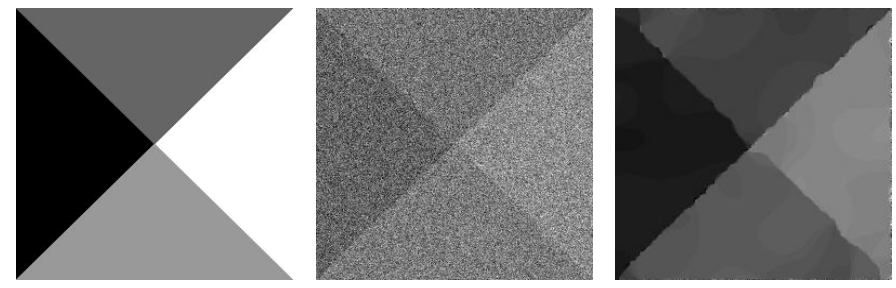

Figure 7.6 - Original, noisy $(\mathrm{SNR}=-7 \mathrm{db}$ ) and reconstructed image, respectively.

\section{Conclusion}

In this work, an automatic PDE model for noise elimination is presented. The two parameters presented in the model are determined using priori information about the standard deviation of the noise we desire to remove and the standard deviation of the noisy image $I$. The relationship between the stop time of the evolution process and the level of the noise elimination desired has been investigated and a new estimative for the optimal smoothing time $T$ for performing the noise elimination in an image was derived.

The main contributions in this paper were given by Theorem 5.1, which gives the value of the stop time for the evolutionary model, and by automatic choice 
of the $k$ parameter given by (6.1). The mathematical analysis shows that the conditions established in the theorem are robust with respect to noise elimination.

The viability of finding the ideal stop evolutionary time for the differential equation avoids computational insufficiency or surplus computation time. The insufficient computation time will not perform good results and the evolution of the differential equations beyond the necessary smoothing time causes an unnecessarily high computational cost. The stop time concept gives an estimative for the time which one should evolve the scale in order to guarantee efficiency and computational gain in the denoising process of an image.

The experimental results show that the scale-space generated by the equation (4.1) using the stop time $T$ as in (5.2) and the constant $k$ in (4.2) calculated by (6.1) gives good signal preservation, when the optimal temporal scale $T$ is reached, maintaining the edges of the image.

\section{REFERENCES}

[1] L. Alvarez, P.L. Lions and J.M. Morel, Image selective smoothing and edge detection by nonlinear diffusion, SIAM J. Numer. Anal., 29 (3) (1992), pp. 845-866.

[2] C.A.Z. Barcelos, M. Boaventura and E.C. Silva Jr., A well-balanced flow equation for noise removal and edge detection, IEEE Trans. on Images Proc., vol. 12 (7) (2003), pp. 751-763.

[3] T. Chan and L. Vese, Variational Image Restoration \& Segmentation Models and Approximations, UCLA report, 47 (1997).

[4] Y. Chen, B.C. Vemuri and L. Wang, Image denoising and Segmentation via nonlinear diffusion, Comput. Math. Appl., 39 (5/6) (2000), pp. 131-149.

[5] L.C. Evans, Convergence of an algorithm for mean curvature motion, Indiana University Mathematics Journal, 42 (1993), pp. 553-557.

[6] J.J. Koenderink, The struture of images, Biol. Cybernet., 50 (1984), pp. 363-370.

[7] J. Malik and P. Perona, Scale-space and edge detection using anisotropic diffusion, IEEE TPAMI, 12 (7) (1990), pp. 629-639.

[8] D. Marr and E. Hildreth, Theory of edge detection, Proc. Roy. Soc. London Ser. B, 207 (1980), pp 187-217.

[9] L. Rudin, S. Osher and E. Fatemi, Nonlinear total Variation based noise removal algorithms, Physica D 60 (1992), pp. 259-268.

[10] J. Shah, A common framework for curve evolution, segmentation and anisotropic diffusion, IEEE Conf. on Computer Vision and Pattern Recognition, June (1996). 
[11] D. Strong and T. Chan, Spatially and scale adaptive total variation based regularization and anisotropic diffusion in image processing, preprint.

[[12]] D.M. Strong, Adaptive Total Variation Minimizing Image Restoration, Ph.D. Thesis, University of California (1997).

[13] C.R. Vogel and M.E. Oman, Iterative methods for total variation denoising, SIAM J. Sci. Statist. Comput., 17 (1996), pp. 227-238.

[14] G. Whitten, Scale Space Tracking and Deformable Sheet Models for Computational Vision, IEEE Transac. Pattern An. Mach. Intell., 15 (7) (1993) pp. 697-706.

[15] A.P. Witkin, Scale-space filtering, Proc. IJCAI, Karlsruhe (1983), pp. 1019-1021. 